\title{
Presurgical Assessment of the Sensorimotor Cortex Using Resting-State fMRI
}

\author{
F.C. Schneider, M. Pailler, I. Faillenot, F. Vassal, J. Guyotat, F.-G. Barral, and IDC. Boutet
}

\begin{abstract}
BACKGROUND AND PURPOSE: The functional characterization of the motor cortex is an important issue in the presurgical evaluation of brain lesions. fMRI noninvasively identifies motor areas while patients are asked to move different body parts. This task-based approach has some drawbacks in clinical settings: long scanning times and exclusion of patients with severe functional or neurologic disabilities and children. Resting-state fMRI can avoid these difficulties because patients do not perform any goal-directed tasks.
\end{abstract}

MATERIALS AND METHODS: Nineteen patients with diverse brain pathologies were prospectively evaluated by using task-based and resting-state $\mathrm{fMRI}$ to localize sensorimotor function. Independent component analyses were performed to generate spatial independent components reflecting functional brain networks or noise. Three radiologists identified the motor components and 3 portions of the motor cortex corresponding to the hand, foot, and face representations. Selected motor independent components were compared with task-based fMRI activation maps resulting from movements of the corresponding body parts.

RESULTS: The motor cortex was successfully and consistently identified by using resting-state fMRI by the 3 radiologists for all patients. When they subdivided the motor cortex into 3 segments, the sensitivities of resting-state and task-based fMRI were comparable. Moreover, we report a good spatial correspondence with the task-based fMRI activity estimates.

CONCLUSIONS: Resting-state fMRI can reliably image sensorimotor function in a clinical preoperative routine. It is a promising opportunity for presurgical localization of sensorimotor function and has the potential to benefit a large number of patients affected by a wide range of pathologies.

ABBREVIATIONS: IC = independent component; ICA = independent component analysis; rs-fMRI = resting-state fMRI; tb-fMRI = task-based fMRI

M apping of cerebral function in neurosurgery patients aims to predict the efficacy of the neurosurgical treatment, estimate the operation risk, and avoid neurologic deficits. Several techniques have been used to identify brain activity in tissue surrounding the regions planned for resection, including neuronavitaged transcranial magnetic stimulation, ${ }^{1}$ magnetoencephalography, ${ }^{2}$ and $\mathrm{fMRI},{ }^{3}$ each having advantages and drawbacks over the others.

Received December 18, 2014; accepted after revision May 29, 2015.

From the Departments of Radiology (F.C.S., M.P., F.-G.B., C.B.), Neurology (I.F.), and Neurosurgery (F.V.), University Hospital of Saint-Etienne, Saint-Etienne, France; Thrombosis Research Group EA 3065 (F.C.S., F.-G.B., C.B.) and Central Integration of Pain Institut National de la Santé et de la Recherche Médicale U1028 (I.F.), Jean Monnet University, Saint-Etienne, France; Image-Guided Clinical Neurosciences and Connectomics EA 7282 (F.V.), Auvergne University, Clermont-Ferrand, France; and Department of Neurosurgery (J.G.), Hospices Civils de Lyon, Claude Bernard University, Lyon, France.

Please address correspondence to Fabien Schneider, PhD, Service de Radiologie, Hôpital Nord, 42055 Saint-Etienne Cedex 2, France; e-mail:

fabien.schneider@univ-st-etienne.fr

http://dx.doi.org/10.3174/ajnr.A4472
The clinical criterion standard for localization of functional brain areas is intraoperative electrical stimulation in the awake patient. ${ }^{4}$ Although electrical stimulation provides unique assistance during surgery, it is an invasive technique that requires expertise of the surgical team and a cooperative and motivated subject. It also adds considerable time to the surgical procedure for an investigation limited to a few cortical areas. Therefore, fMRI has been seen as very promising for clinical applications. However, its integration into preoperative surgical planning has been relatively slow because of several practical constraints: the dedicated experimental setup, long scanning time, and a high cognitive demand on the patient. Moreover, localizing the sensorimotor cortex with fMRI at the individual level can be challenging in some cases when the patient has paresis or paralysis. Furthermore, $>1$ acquisition is necessary whenever the lesion is bordering on several motor representations.

Techniques measuring functional connectivity can address several of the limitations faced by stimulus-driven or task-based fMRI (tb-fMRI). Resting-state fMRI (rs-fMRI) uses slow, sponta- 
Table 1: Patient characteristics

\begin{tabular}{|c|c|c|c|c|c|c|}
\hline Case & Age (yr) & Sex & Diagnosis & Localization & Lateralization & tb-fMRI \\
\hline 1 & 67 & $M$ & 3 Metastases & SFG & $2 \mathrm{R}, 1 \mathrm{~L}$ & $\mathrm{RH}, \mathrm{RF}$, face \\
\hline 2 & 28 & $\mathrm{~F}$ & Low-grade glioma & SFG & L & $\mathrm{RH}$, face \\
\hline 3 & 39 & M & Low-grade glioma & RG & L & $\mathrm{RH}$ \\
\hline 4 & 53 & M & AVM & IFG, insula & $\mathrm{R}$ & LH, face \\
\hline 5 & 28 & $M$ & AVM & FG & L & Face \\
\hline 6 & 43 & $\mathrm{~F}$ & Stroke & $\mathrm{PP}$ & $\mathrm{R}$ & $\mathrm{RH}, \mathrm{LH}, \mathrm{RF}, \mathrm{LF}$, face \\
\hline 7 & 45 & M & High-grade glioma & SPL & L & $\mathrm{RH}, \mathrm{LH}, \mathrm{RF}$ \\
\hline 8 & 50 & M & Stroke & IOG, cerebellum & $R$ & RH, LH, LF, face \\
\hline 9 & 12 & $M$ & Low-grade glioma & SFG, PrG & L & $\mathrm{RH}, \mathrm{LH}, \mathrm{RF}, \mathrm{LF}$ \\
\hline 10 & 66 & $M$ & Metastasis & PoG & $\mathrm{R}$ & $\mathrm{RH}, \mathrm{LH}, \mathrm{LF}$ \\
\hline 11 & 58 & $M$ & Stroke & Brain stem & L & RH, LH, RF, face \\
\hline 12 & 68 & M & Stroke & Brain stem & $\mathrm{R}$ & $\mathrm{RH}, \mathrm{LH}, \mathrm{LF}$, face \\
\hline 13 & 26 & $F$ & Low-grade glioma & SFG, CG & $\mathrm{R}$ & Face \\
\hline 14 & 77 & M & Metastasis & PoG & $\mathrm{R}$ & $\mathrm{RH}, \mathrm{LH}$ \\
\hline 15 & 70 & $\mathrm{~F}$ & Metastasis & PoG & $\mathrm{L}$ & $\mathrm{RH}$ \\
\hline 16 & 58 & $M$ & Low-grade glioma & PrG, PoG & $R$ & LH, LF \\
\hline 17 & 28 & M & Low-grade glioma & SFG & L & $\mathrm{RH}, \mathrm{LH}$ \\
\hline 18 & 31 & $\mathrm{~F}$ & Low-grade glioma & SFG & $R$ & $\mathrm{RH}, \mathrm{LH}$ \\
\hline 19 & 23 & M & Low-grade glioma & SFG, PrG & $\mathrm{R}$ & $\mathrm{RH}, \mathrm{LH}$ \\
\hline
\end{tabular}

Note:-L indicates left; R, right; RH, right hand; LH, left hand; RF, right foot; LF, left foot; SFG, superior frontal gyrus; RG, rectus gyrus; IFG, inferior frontal gyrus; FG, fusiform gyrus; PP, periventricular parietal white matter; SPL, superior parietal lobule; IOG, inferior occipital gyrus; PrG, precentral gyrus; PoG, postcentral gyrus; CG, cingulate gyrus.

neous fluctuations in the blood oxygen level-dependent signal to characterize networks of distant brain regions. ${ }^{5}$ The subject simply "rests" in the scanner without any specific task to perform. rs-fMRI has been successfully applied in groups of healthy volunteers, ${ }^{6}$ provides a means of mapping several functional networks in a single acquisition, appears robust across individuals, ${ }^{7}$ and is less-demanding than tb-fMRI because it requires less cooperation from the patient and can be used in individuals with neurologic deficits or cognitive dysfunction or in children. Additionally, spontaneous activity continues in the primary sensory and motor cortices even when subjects are asleep ${ }^{8}$ or anesthetized. ${ }^{9}$ This feature suggests that complete patient compliance may not be necessary. Resting-state networks have been extensively explored in recent years at the group level in populations of healthy subjects and patients. In the case of neurosurgery patients with brain damage, ${ }^{10}$ precise functional network estimation at the individual level is essential for surgery planning and/or intraoperative navigation. The conclusions of previous pioneering studies were limited to the feasibility of rs-fMRI for presurgical mapping by using small patient samples. ${ }^{11-15}$

In this study, we investigated the sensitivity of extracting the sensorimotor network from rs-fMRI at the individual level in patients with brain damage scheduled for surgery. Because brain lesions can appear at any segment of the motor cortex, we divided our investigations into 3 portions of the somatotypy (foot, hand, and face). rs-fMRI was then compared with tb-fMRI acquired when corresponding body parts were moved.

\section{MATERIALS AND METHODS \\ Subjects}

Nineteen patients (14 men; mean age, 46 years) intended for neurosurgery were prospectively enrolled in this study (Table 1) and underwent both tb- and rs-fMRI. tb-fMRI results were used for surgical planning. Pathologies included low-grade glioma $(n=$ $8)$, high-grade glioma $(n=1)$, metastasis $(n=4)$, AVM $(n=2)$, and chronic stroke lesions $(n=4)$ causing medically refractory neuropathic pain (stimulator positioning was guided by tb-fMRI for chronic electrical stimulation of the motor cortex). Patients gave written informed consent, and the study was approved by the local ethics committee.

\section{Image Acquisition}

Nine patients (cases $1-6,8,16,17$ ) were imaged by using a $1.5 \mathrm{~T}$ MR imaging scanner (Achieva; Philips Healthcare, Best, the Netherlands) equipped with an 8-channel head coil. Single-shot gradient-echo EPI was used for functional acquisitions with the following parameters: TR/TE/flip angle, $2700 \mathrm{~ms} / 50 \mathrm{~ms} / 90^{\circ}, 28$ contiguous sections of $3.5 \mathrm{~mm}$, FOV of $230 \times 230 \mathrm{~mm}^{2}$, and an acquisition matrix of $64 \times 64 ; 105$ dynamic scans per run were acquired for tb-fMRI acquisitions, and 130, for rs-fMRI. A 3D T1-weighted turbo field echo sequence was performed for anatomic localization: TR/TE/flip angle, $7.84 \mathrm{~ms} / 3.81 \mathrm{~ms} / 8^{\circ}, 140 \mathrm{sec}-$ tions of $1 \mathrm{~mm}$, acquisition matrix of $256 \times 256$, FOV of $256 \times 256$ $\mathrm{mm}^{2}$, and a turbo field echo factor of 180 .

The images of the 10 remaining patients were acquired by using a 3T scanner (Magnetom Verio; Siemens, Erlangen, Germany) and a 12-channel head coil. Single-shot gradient-echo EPI parameters were the following: TR/TE/flip angle, $2690 \mathrm{~ms} / 30 \mathrm{~ms} /$ $90^{\circ}, 40$ contiguous sections of $3.5 \mathrm{~mm}$, FOV of $224 \times 224 \mathrm{~mm}^{2}$, and an acquisition matrix of $64 \times 64$. tb-fMRI comprised 110 dynamic scans per run and rs-fMRI, 155. A 3D T1-weighted magnetization-prepared rapid acquisition of gradient echo sequence was also performed with the following parameters: TR/TI/TE/flip angle, $1800 \mathrm{~ms} / 900 \mathrm{~ms} / 3.3 \mathrm{~ms} / 9^{\circ}$, acquisition matrix of $256 \times$ 192 , FOV of $256 \times 192 \mathrm{~mm}^{2}$.

\section{Behavioral Paradigm}

For rs-fMRI, subjects were instructed to stay quiet and fixate on a white cross on a black screen. The tb-fMRI examination included 1-5 motor runs, each containing only 1 type of movement (which involved either the right/left hand, right/left foot, or face). Hand movements were self-paced hand tapping. Face movements consisted of stretching and pushing forward the lips. Foot movements included rotations of the ankle (dorsiflexion-extension). Each tb- 
fMRI acquisition consisted of 30-second blocks of motor movement alternating with 30 -second blocks of resting.

\section{Data Preprocessing}

The 3 initial brain volumes of each run were discarded to eliminate nonequilibrium effects of magnetization. Image preprocessing was performed with SPM8 (http://www.fil.ion.ucl.ac.uk/spm/software/ spm8). Images were time- and motion-corrected and then smoothed by using a spatial Gaussian kernel of $8 \mathrm{~mm}$ of full width at half maximum.

\section{Independent Component Analysis of rs-fMRI}

To investigate resting-state networks for each patient, we performed retrospective analyses of single-subject independent component analysis (ICA) by using the Group ICA of fMRI Toolbox software (GIFT; http://mialab.mrn.org/software/gift/). The number of independent components (ICs) was estimated individually for each subject by using the minimum description length criteria. ${ }^{16}$ Before the ICA procedure, a data-reduction step was performed by using principal component analysis. The ICs were then estimated by using the Infomax algorithm. ${ }^{17}$ Stable estimation was achieved by rerunning the ICA analyses 20 times by using the ICASSO ${ }^{18}$ toolbox implemented in GIFT. In case of ICs with an ICASSO stability index of $<0.9$, the ICA analysis was recomputed by using fewer ICs (the number of ICs estimated by minimum description length minus the number of ICs with an ICASSO stability index of $\leq 0.9$ ). Eighteen-to-34 ICs were then estimated in our patient sample. The spatial maps of the components were converted into $z$ score maps and thresholded at $z \geq 2$. The mean image of the rs-fMRI timeseries (estimated during the motion-correction procedure) was used to coregister the $z$ score maps on the anatomic MR imaging acquisition to allow a precise spatial localization of the resting-state networks by using SPM8.

\section{Manual Selection of Individual Motor Components by Experts}

For each patient, 3 independent experts (C.B., F.C.S., and M.P.), blinded to tb-fMRI results, visualized all thresholded ICs superimposed on T1 images and selected any number of motor-related components. Of note, F.C.S. participated in both the prospective interpretation of 13 patients explored with tb-fMRI and the retrospective readings of the rs-fMRI. The time interval between the 2 tasks was 2 months for 2 patients and $>1$ year for 17 patients. The IC selection criteria were based on the shape of spatial maps, temporal profiles, and anatomic landmarks (eg, central sulcus). The central sulcus was reported as difficult or impossible to detect in 6 cases, either because of the presence of extensive edema (cases 1,10 , and 16) or the tumor itself (cases 9, 14, and 15). Readers were also asked to locate IC clusters within the motor cortex and whether they could be attributed to the foot, the hand, or the face representation.

In the case of different motor IC selection among the experts, a consensus was reached after consultation to select which IC most likely reflects the motor cortex for both hemispheres.

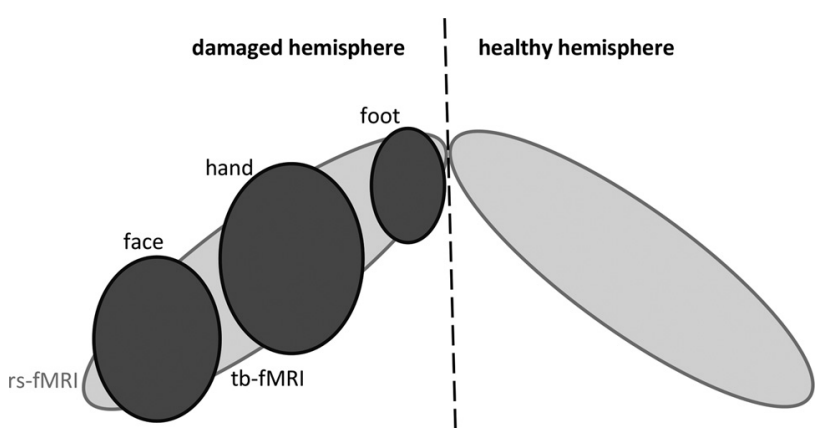

FIG 1. Illustration of expected findings by using rs-fMRI (light gray) and tb-fMRI (dark gray). rs-fMRI is presumed to show the whole motor cortex (possibly bilaterally), whereas a single motor cortex representation (face, hand, or foot) would be obtained by using tb-fMRI.

\section{General Linear Modeling of t $b-f M R I$}

The tb-fMRI data were high-pass-filtered $(1 / 128 \mathrm{~Hz})$ and analyzed through the general linear formulation of SPM8. The model also included the 6 movement parameters estimated from the motion-correction procedure to capture residual movement-related artifacts. Statistical parametric maps were thresholded at $P<.05$, corrected for multiple comparisons (controlling for the family-wise error) by using 2 different procedures: The first method tested each voxel time course (called the "voxel" correction), and the second combined the spatial extent of each cluster of estimated activity and each voxel intensity (termed hereafter "cluster," 19 ). The mean image of the tb-fMRI dataset was used to coregister the activation maps on the anatomic MR imaging acquisition to allow a precise localization of the activated areas and a direct comparison with rs-fMRI results (see below).

\section{tb-fMRI and rs-fMRI Sensitivities}

The sensitivity of rs-fMRI was estimated by dividing the number of times a motor cortex representation (hand, face, or foot) was detected by the total number of patients. The sensitivity of tb-fMRI was calculated as the ratio of detections for a given motor cortex representation (hand, face, or foot) by the number of patients for whom this investigation was performed (Table 1).

\section{Overlap between rs-fMRI and tb-fMRI}

To reduce the influence of voxels outside the motor cortex on the results, we segmented both rs- and tb-fMRI spatial maps around the cluster defined by experts as the motor cortex (spm clusters function; http://www.fil.ion.ucl.ac.uk/spm/software/ spm8/). The result of this procedure gave a single cluster for the motor IC and a single cluster for tb-fMRI (called rs-motor and tb-motor). Then, we calculated a concurrence ratio by dividing the number of voxels within the intersection of rs- and tb-motor by the number of voxels of tb-motor (Figs 1 and 2).

\section{RESULTS}

Nine patients had lesions in the left hemisphere. For consistency of the data presentation, we hereafter refer to healthy and damaged sides. For 1 patient, metastases were detected in both hemispheres (case 1). The left side was then defined as the altered one because the corresponding tumor was considered 
the most likely to have caused the patient deficits (because of its location, size, and edema). We tested the motoricity of the hand for 17 patients, the foot for 9 patients, and the face for 9 patients (Table 1). Figure 2 illustrates typical rs- and tb-fMRI results.

\section{tb-fMRI and rs-fMRI Sensitivities}

The rs-motor component was identified for all patients and by all experts. A consensus was reached in all patients for the hand area, in 15 patients for the face area, and in 13 for the foot representation (Table 2). tb-fMRI investigations led to significant activity

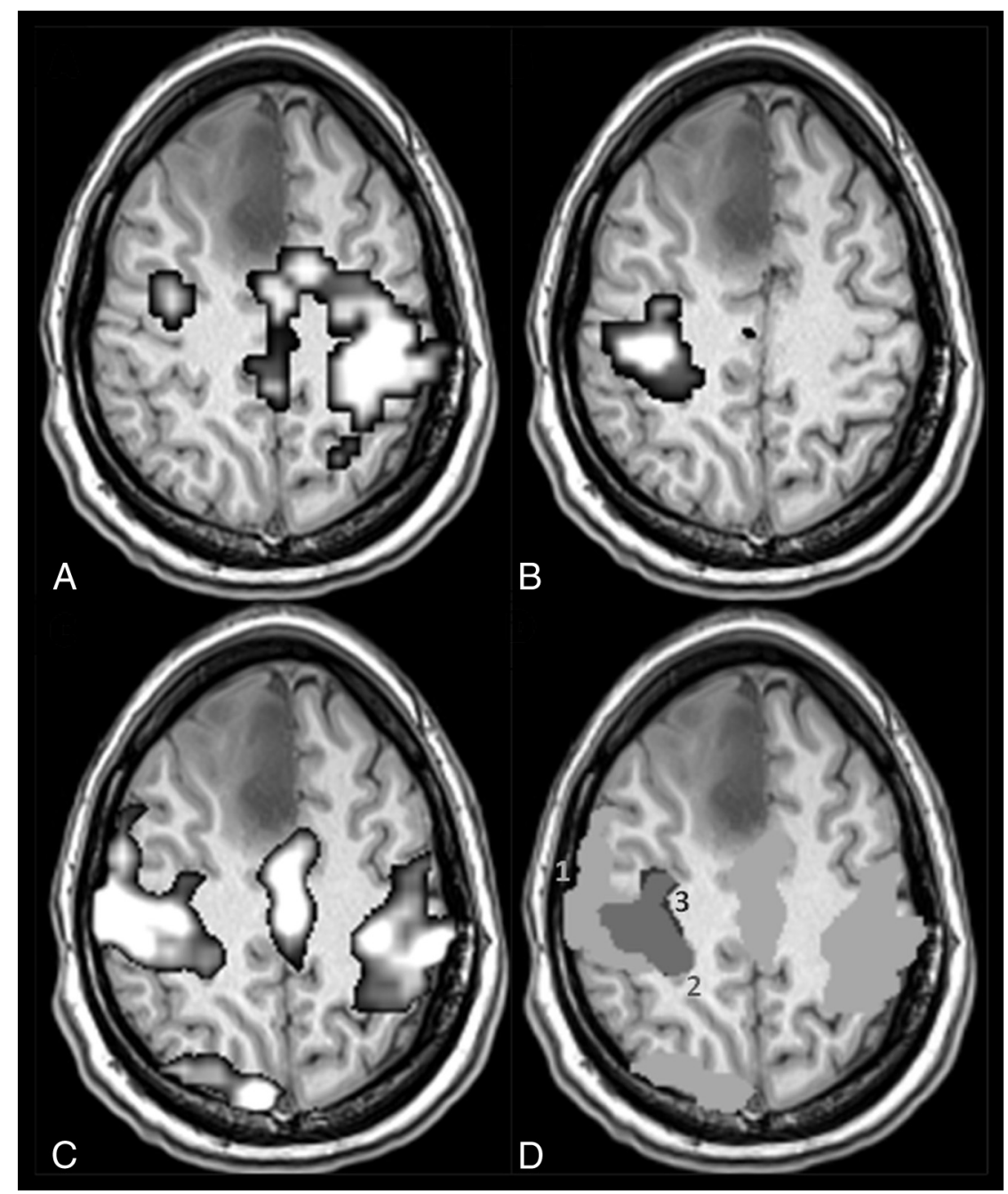

FIG 2. A patient with a right medial frontal low-grade glioma (case 18 in Table 1). Activity estimates from right $(A)$ and left $(B)$ hand movements by using tb-fMRI $(P<.05$, family-wise error-corrected at the voxel level) are shown and are compared with the motor IC of rs-fMRI $(C, z \geq 2)$. The overlap between rs-and tb-fMRI results is illustrated in $D$, where spatial maps of $B$ and $C$ are superimposed after binarization and segmentation of the motor clusters (see "Materials and Methods"): 1 (light gray) and 3 (black), respectively, indicate rs- and tb-spatial maps, and their intersection is labeled 2 (dark gray). The concurrent ratio is $92 \%$ for this patient. estimates, in most cases independent of the thresholding strategy (voxel or cluster).

\section{Overlap between rs-fMRI and tb-fMRI}

We did not observe any significant interactions among the hand, face, or foot representations and the overlap between rs- and tbfMRI (Fig 3). Moreover, their similarity did not significantly differ between the 2 hemispheres. In contrast, we observed a better correspondence between rs- and tb-fMRI when using the voxel correction for multiple testing ( $31 \%$ versus $23 \%, P=.003$ ). This advantage was more pronounced on the lesion side $(P=.009)$ and for the hand representation $(P=.01)$.

\section{Comparison of the 2 MR Imaging Acquisition Systems}

The overall rs-fMRI sensitivity of the 3T system was higher than that for the $1.5 \mathrm{~T}$ system $(P=.0029$, Table 3$)$. Moreover, the intersection between tb- and rsfMRI results did not differ significantly between the 2 MR imaging systems, though there was a trend toward higher concurrent ratios at $3 \mathrm{~T}$ when using both the voxel ( $35 \%$ versus $27 \%, P=.33$ ) or the cluster correction (27\% versus $20 \%$, $P=.29)$ for multiple testing of tb-fMRI statistical maps.

\section{DISCUSSION}

Presurgical localization of the eloquent cortex is clearly one of the keys to reducing postsurgery motor deficits and reaching an optimal patient outcome. Among the functional imaging techniques, rs-fMRI has been commonly used in neuroscience and has now attracted interest for clinical applications. $^{20,21}$ Compared with tb-fMRI, rsfMRI has some practical advantages: shorter scanning times and a simple setup. It is less sensitive to subject-related deficiencies such as paresis, paralysis, or lack of attention, and it is easier to apply to children. Here, we demonstrated that rs-fMRI is capable of consistently mapping the motor cortex with a spatial precision that is necessary in clinical settings (ie, discrimination of foot, hand, and face representations). The probability of identifying different body representations by using rs-fMRI is

Table 2: Sensitivity of rs- and tb-fMRI

\begin{tabular}{|c|c|c|c|c|c|c|}
\hline & \multicolumn{2}{|c|}{ Foot } & \multicolumn{2}{|c|}{ Hand } & \multicolumn{2}{|c|}{ Face } \\
\hline & Healthy Side & Damaged Side & Healthy Side & Damaged Side & Healthy Side & Damaged Side \\
\hline rs-fMRI & $68 \%(13 / 19)$ & $68 \%(13 / 19)$ & $100 \%(19 / 19)$ & $100 \%(19 / 19)$ & $79 \%(15 / 19)$ & $79 \%(15 / 19)$ \\
\hline tb-fMRI cluster-correction & $50 \%(1 / 2)$ & $89 \%(8 / 9)$ & $100 \%(11 / 11)$ & $94 \%(16 / 17)$ & $100 \%(9 / 9)$ & $100 \%(9 / 9)$ \\
\hline tb-fMRI voxel-correction & $50 \%(1 / 2)$ & $89 \%(8 / 9)$ & 100\% (11/11) & $94 \%(16 / 17)$ & $89 \%(8 / 9)$ & $89 \%(8 / 9)$ \\
\hline
\end{tabular}


equivalent to that of tb-fMRI. Nevertheless, unequal results were obtained in different parts of the motor cortex: The largest body part representation (hand) was the easiest to detect and the most consistent among readers (Table 2). Our findings are in line with those of Liu et al, ${ }^{13}$ who studied 6 patients with tumors or epileptic foci near the motor cortex before surgery. The visual evaluation of their results led to good agreement between rs-fMRI and tb-fMRI, which included hand and tongue movements. We also measured the overlap between rs- and tb-fMRI through concurrent ratios. The average value was approximately $30 \%$, which is in agreement with the findings of Tie et $\mathrm{al}^{22}{ }^{22}$ who assessed concurrent ratios in healthy individuals.

Of several methods that can be used to analyze tb-fMRI data, ${ }^{23}$ we used SPM, and dissimilar results may be obtained with other software. Particularly, different strategies can be applied to deal with the multiple-testing problem when analyzing tb-fMRI data. $^{24,25}$ No consensus has been reached on which one to use in the context of individual patient analysis. We investigated 2 dif-
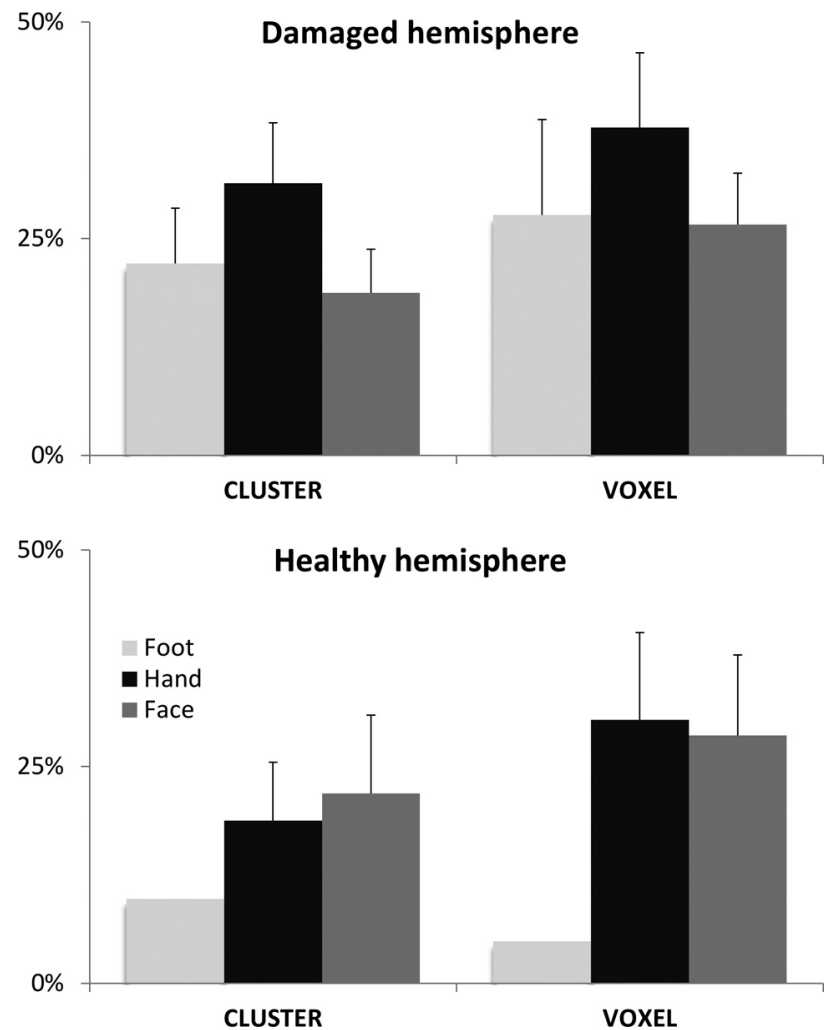

FIG 3. Overlap between tb-fMRI and rs-fMRI. Average (and standard error of the mean) concurrent ratios are displayed for the damaged hemisphere and the healthy hemisphere. Different body parts were moved during tb-fMRI, leading to 3 ratio calculations: foot (light gray), hand (black), and face (dark gray). Two different thresholding strategies were tested for tb-fMRI analysis: a test based on the cluster size (cluster) and another one based on the voxel intensity (voxel).

Table 3: Sensitivity of rs-fMRI for the 2 MR imaging systems

\begin{tabular}{|c|c|c|c|c|c|c|}
\hline & \multicolumn{2}{|c|}{ Foot } & \multicolumn{2}{|c|}{ Hand } & \multicolumn{2}{|c|}{ Face } \\
\hline & $\begin{array}{l}\text { Healthy } \\
\text { Side }\end{array}$ & $\begin{array}{l}\text { Damaged } \\
\text { Side }\end{array}$ & $\begin{array}{l}\text { Healthy } \\
\text { Side }\end{array}$ & $\begin{array}{l}\text { Damaged } \\
\text { Side }\end{array}$ & $\begin{array}{l}\text { Healthy } \\
\text { Side }\end{array}$ & $\begin{array}{c}\text { Damaged } \\
\text { Side }\end{array}$ \\
\hline $1.5 \mathrm{~T}$ & $55 \%(5 / 9)$ & $55 \%(5 / 9)$ & $100 \%(9 / 9)$ & $100 \%(9 / 9)$ & $67 \%(6 / 9)$ & $67 \%(6 / 9)$ \\
\hline $3 \mathrm{~T}$ & $80 \%(8 / 10)$ & $80 \%(8 / 10)$ & $100 \%(10 / 10)$ & $100 \%(10 / 10)$ & $90 \%(9 / 10)$ & $90 \%(9 / 10)$ \\
\hline
\end{tabular}

ferent approaches to correct for multiple comparisons in our work: a test based on the cluster size and another based on voxel intensity. The cluster size correction is known to have increased sensitivity when the signal is spatially extended ${ }^{19,25}$; this result is likely when attempting to detect the activity of the sensorimotor cortex. The sensitivity of both tests was similar and approximately $95 \%$ in our data (Table 2). However, when comparing tb- and rs-fMRI spatial maps, we observed different overlap depending on the multiple-comparison technique; concurrent ratios were larger with the correction at the voxel level.

Of note, both the rs-ICs and the tb-activation maps did not exclusively show the motor cortex. tb-fMRI revealed a somatotopic segment of the motor cortex and can display nonspecific activations. rs-fMRI motor components showed the whole motor cortex and were typically bilateral (Fig 1). To limit the impact of this problem, we segmented the spatial maps resulting from rsand tb-fMRI analyses. However, the outcome usually showed regions larger than the primary motor cortex (Fig 2). This effect frequently occurred when using the cluster correction because it is more likely to expose large clusters of activity and may explain the lower concurrent ratios obtained when using this test.

Most previous studies testing rs-fMRI clinical feasibility used cross-correlation techniques. ${ }^{11,13-15}$ This approach requires the definition of a seed region based on anatomy or additional functional exploration, to reveal correlations between the average signal time course of voxels within the ROI and the time courses of the other brain voxels. The approach we used here (ICA) does not require any prior knowledge of the temporal or spatial patterns of brain responses. ICA is a popular mathematic approach that maximizes statistical independence among its components to identify distinct resting-state networks. A practical drawback that can be attributed to ICA is that the user must choose among dozens of functional components, which most probably reflect neurofunctional systems over noise. This selection is often made by visual inspection. Other strategies have been imagined to assist the IC selection. Kokkonen et $\mathrm{al}^{12}$ used a template-matching method, for which each IC was spatially correlated to a motor tb-fMRI template. The authors reported successful results in 8 patients with brain tumors. Nevertheless, to avoid adding any prior spatial knowledge in our analyses, we chose another strategy to analyze our rs-fMRI data. Visual review of the calculated ICs for every patient was thus made by 3 radiologists blinded to each other and to the results of tb-fMRI. Additionally, a novel data-driven method has been recently tested at the individual level in patients with epilepsy and tumors. ${ }^{26}$ It showed promising results and may resolve some of the limitations of cross-correlation analysis (structural seed definition) and ICA (postanalysis IC selection).

A tb-fMRI examination including movement of the hand, face, and foot gives satisfactory coverage of the motor cortex for many clinical purposes. The number of studied body parts has to be adapted to the lesion location. Nonetheless, when considering a large lesion, one must examine the entire motor cortex of the injured hemisphere and one may also be interested in mapping the normal motor representation in the contralateral hemisphere. This process leads to long scanning times and may not be adapted to every 
patient, especially when the functional exploration is combined with morphologic and/or spectroscopic acquisitions.

Case 9 relates to a 12-year-old child with a glioma, for whom the whole MR imaging examination may have been too long and too demanding to be entirely and satisfactorily completed. Indeed, the tb-fMRI explorations of both feet did not give any significant results. Alternatively, both complete motor cortices were then identified by using rs-fMRI in $<7$ minutes. Because a long scanning time is the most frequent difficulty for the schedule of tb-fMRI examinations, rs-fMRI is a convincing surrogate and can be more easily integrated with other MR imaging investigations. For another case (15), the patient had difficulty performing the task because of a Dupuytren contracture. This led to nonsignificant and uninterpretable results by using tb-fMRI. This case illustrates the limits of tb-fMRI in a clinical environment where it is difficult to design a specific task for each patient. In contrast, rs-fMRI results were informative.

Additionally, we showed that rs-fMRI can be successfully performed by using 2 different MR imaging systems (1.5T and 3T). Nevertheless, we report a better ability to detect the motor cortex at 3T and a trend toward a better similarity between rs-and tbfMRI spatial maps at 3T (Table 3). Indeed, the spatial specificity of tb-fMRI increases with the strength of the magnetic field. ${ }^{27}$ Moreover, because the amplitude of spontaneous blood oxygen leveldependent fluctuations is also likely to increase with field strength, it is then not surprising to find a better intersection between rs- and tb-fMRI results at 3T.

Generalizability in real-life clinical settings is usually a critical step when developing new techniques. Here, we successfully tested rs-fMRI in a prospective study, involving a typical patient list of our department by using 2 different clinical MR imaging systems. Sensitivity was comparable with that of tb-fMRI with fewer practical acquisition issues. The IC selection process still needs to be standardized and automated in some way to allow rs-fMRI to be largely used for clinical planning before surgery. Moreover, preliminary studies characterizing the accuracy of rsfMRI showed promising results. ${ }^{15,26}$ These findings constitute the groundwork for the establishment of rs-fMRI in surgical planning. However, improved patient outcome has yet to be demonstrated, to firmly establish the clinical utility of rs-fMRI.

\section{CONCLUSIONS}

We showed that rs-fMRI can reliably image the sensorimotor function in preoperative routine. Different motor cortex representations were successfully and consistently identified by 3 radiologists. Moreover, we report good spatial correspondence with tb-fMRI activity estimates. rs-fMRI is a promising opportunity for presurgical localization of sensorimotor function and can resolve most of the tb-fMRI issues in clinical settings. Moreover, rs-fMRI can be easily integrated with other MR imaging investigations. Our study brings new advances in the clinical utility of rs-fMRI.

Disclosures: Fabrice-Guy Barral—RELATED: Support for Travel to Meetings for the Study or Other Purposes: ALN Compagny, ${ }^{*}$ Comments: Cardiovascular and Interventional Radiological Society European Congress 2014. Claire Boutet-UNRELATED: Payment for Development of Educational Presentations: Boehringer Ingelheim, Comments: 300 euros for a teaching communication for 1 day on stroke imaging;
Travel/Accommodations/Meeting Expenses Unrelated to Activities Listed: Guerbet, Ipsen Pharma, Biogen, St Jude Medical France, Toshiba, GE Medical System and Genzyme, Comments: payment directly of accommodation and transport for my participation in a national congress. *Money paid to the institution.

\section{REFERENCES}

1. Weiss C, Tursunova I, Neuschmelting V, et al. Improved nTMS- and DTI-derived CST tractography through anatomical ROI seeding on anterior pontine level compared to internal capsule. Neuroimage Clin 2015;7:424-37 CrossRef Medline

2. Stufflebeam SM. Clinical magnetoencephalography for neurosurgery. Neurosurg Clin N Am 2011:22:153-67, vii-viii

3. Mueller WM, Yetkin FZ, Hammeke TA, et al. Functional magnetic resonance imaging mapping of the motor cortex in patients with cerebral tumors. Neurosurgery 1996;93:515-20; discussion 520-21

4. Haglund MM, Berger MS, Shamseldin M, et al. Cortical localization of temporal lobe language sites in patients with gliomas. Neurosurgery 1994;34:567-76; discussion 576 CrossRef Medline

5. Fox MD, Raichle ME. Spontaneous fluctuations in brain activity observed with functional magnetic resonance imaging. Nat Rev Neurosci 2007;8:700-11 CrossRef Medline

6. Auer DP. Spontaneous low-frequency blood oxygenation level-dependent fluctuations and functional connectivity analysis of the “resting” brain. Magn Reson Imaging 2008;26:1055-64 CrossRef Medline

7. Damoiseaux JS, Rombouts SA, Barkhof F, et al. Consistent restingstate networks across healthy subjects. Proc Natl Acad Sci U S A 2006;103:13848-53 CrossRef Medline

8. Fukunaga M, Horovitz SG, van Gelderen P, et al. Large-amplitude, spatially correlated fluctuations in BOLD fMRI signals during extended rest and early sleep stages. Magn Reson Imaging 2006;24: 979-92 CrossRef Medline

9. Kiviniemi VJ, Haanpää H, Kantola JH, et al. Midazolam sedation increases fluctuation and synchrony of the resting brain BOLD signal. Magn Reson Imaging 2005;23:531-37 CrossRef Medline

10. Holodny AI, Schulder M, Liu WC, et al. The effect of brain tumors on BOLD functional MR imaging activation in the adjacent motor cortex: implications for image-guided neurosurgery. AJNR Am J Neuroradiol 2000;21:1415-22 Medline

11. Böttger J, Margulies DS, Horn P, et al. A software tool for interactive exploration of intrinsic functional connectivity opens new perspectives for brain surgery. Acta Neurochir (Wien) 2011;153:1561-72 CrossRef Medline

12. Kokkonen SM, Nikkinen J, Remes J, et al. Preoperative localization of the sensorimotor area using independent component analysis of resting-state fMRI. Magn Reson Imaging 2009;27:733-40 CrossRef Medline

13. Liu H, Buckner RL, Talukdar T, et al. Task-free presurgical mapping using functional magnetic resonance imaging intrinsic activity. J Neurosurg 2009;111:746-54 CrossRef Medline

14. Quigley M, Cordes D, Wendt G, et al. Effect of focal and nonfocal cerebral lesions on functional connectivity studied with MR imaging. AJNR Am J Neuroradiol 2001;22:294-300 Medline

15. Zhang D, Johnston JM, Fox MD, et al. Preoperative sensorimotor mapping in brain tumor patients using spontaneous fluctuations in neuronal activity imaged with functional magnetic resonance imaging: initial experience. Neurosurgery 2009:5(6 suppl):226-36 CrossRef Medline

16. Li YO, Adali T, Calhoun VD. Estimating the number of independent components for functional magnetic resonance imaging data. Hum Brain Mapp 2007;28:1251-66 CrossRef Medline

17. Bell AJ, Sejnowski TJ. An information-maximization approach to blind separation and blind deconvolution. Neural Comput 1995;7: 1129-59 CrossRef Medline

18. Himberg J, Hyvärinen A, Esposito F. Validating the independent components of neuroimaging time series via clustering and visualization. Neuroimage 2004;22:1214-22 CrossRef Medline 
19. Poline JB, Worsley KJ, Evans AC, et al. Combining spatial extent and peak intensity to test for activations in functional imaging. Neuroimage 1997;5:83-96 CrossRef Medline

20. Castellanos FX, Di Martino A, Craddock RC, et al. Clinical applications of the functional connectome. Neuroimage 2013;80:527-40 CrossRef Medline

21. Lee MH, Smyser CD, Shimony JS. Resting-state fMRI: a review of methods and clinical applications. AJNR Am J Neuroradiol 2013;34: 1866-72 CrossRef Medline

22. Tie $\mathrm{Y}$, Rigolo L, Norton IH, et al. Defining language networks from resting-state fMRI for surgical planning: a feasibility study. Hum Brain Mapp 2014;3:1018-30 CrossRef Medline

23. Carp J. The secret lives of experiments: methods reporting in the fMRI literature. Neuroimage 2012;63:289-300 CrossRef Medline
24. Nichols TE. Multiple testing corrections, nonparametric methods, and random field theory. Neuroimage 2012;62:811-15 CrossRef Medline

25. Hayasaka S, Nichols TE. Validating cluster size inference: random field and permutation methods. Neuroimage 2003;20:2343-56 CrossRef Medline

26. Mitchell TJ, Hacker CD, Breshears JD, et al. A novel data-driven approach to preoperative mapping of functional cortex using resting-state functional magnetic resonance imaging. Neurosurgery 2013;73:969-82; discussion 982-83 CrossRef Medline

27. Ogawa S, Menon RS, Tank DW, et al. Functional brain mapping by blood oxygenation level-dependent contrast magnetic resonance imaging: a comparison of signal characteristics with a biophysical model. Biophys J 1993;64:803-12 CrossRef Medline 